Pacific Journal of Mathematics

VOLUME ESTIMATES FOR REAL HYPERSURFACES OF 


\section{VOLUME ESTIMATES FOR REAL HYPERSURFACES OF A KAEHLER MANIFOLD WITH STRICTLY POSITIVE HOLOMORPHIC SECTIONAL AND ANTIHOLOMORPHIC RICCI CURVATURES}

\section{FERNANDO GiménEZ ANd Vicente MiQUel}

We give some inequalities for the volume of a connected compact real hypersurface of a compact Kaehler manifold with strictly positive holomorphic sectional and antiholomorphic Ricci curvatures and prove that some of the corresponding equalities characterize the geodesic spheres in $C P^{n}(\lambda)$.

1. Introduction. Let $M$ be an $n$-dimensional connected compact Riemannian manifold $M$. Let $P$ be a connected compact hypersurface of $M$. Suppose that the Ricci curvature of $M$ is bounded from below by a real number $(n-1) \lambda, \lambda>0$. Let $\Lambda$ be an upper bound of the norm of the mean curvature of $P$. Heintze and Karcher ([H-K]) proved that the following inequality holds:

$$
\operatorname{vol}(M) \leq \frac{\operatorname{vol}\left(S_{\lambda}^{n}\right)}{\operatorname{vol}\left(S_{\lambda+\Lambda^{2}}^{n-1}\right)} \operatorname{vol}(P),
$$

where $S_{k}^{q}$ denotes the $q$-sphere of constant sectional curvature $k$.

Moreover they showed that equality in (1.1) implies that both $M$ and $P$ are of constant curvature.

Observe that $S_{\lambda+\Lambda^{2}}^{n-1}$ is isometric to a geodesic sphere of $S_{\lambda}^{n}$ of radius $r$ given by

$$
\frac{1}{\sqrt{\lambda+\Lambda^{2}}}=\frac{1}{\sqrt{\lambda}} \sin (\sqrt{\lambda} r), \quad 0 \leq r \leq \frac{\pi}{\sqrt{\lambda}} .
$$

Let $\mathscr{R}_{n, \lambda, \Lambda}$ be the family of pairs $(P, M)$ of connected compact Riemannian manifolds satisfying the hypothesis in the above statement of the Heintze-Karcher's result. Let us consider the function "relative volume" $\mathscr{V}: \mathscr{R}_{n, \lambda, \Lambda} \rightarrow \mathbb{R}$ defined by $\mathscr{V}(P, M)=(\operatorname{vol}(P)) /(\operatorname{vol}(M))$. Then Heintze-Karcher's theorem is equivalent to: "For every triad $(n, \lambda, \Lambda)$, the function $\mathscr{V}$ defined on $\mathscr{R}_{n, \lambda, \Lambda}$ has a minimum $C(n, \lambda, \Lambda)$ $=\mathscr{V}\left(S_{\lambda+\Lambda^{2}}^{n-1}, S_{\lambda}^{n}\right)$, and this is the only pair on which the minimum is attained". 
Then it seems to be interesting to find subsets $\mathscr{B} \subset \mathscr{R}_{n, \lambda, \Lambda}$ on which there is a lower bound $B$ of $\mathscr{V}, B>C(n, \lambda, \Lambda)$, to study if the bound $B$ is a minimum of $\mathscr{V}$ on $\mathscr{B}$ and, if this is the case, to know the elements in $\mathscr{B}$ realizing this minimum. We look for these subsets $\mathscr{B}$ in $\mathscr{R}_{n, \lambda, \Lambda} \cap \mathscr{K}_{n}$, where $\mathscr{K}_{n}$ is the family of pairs $(P, M)$ such that $M$ is a connected compact Kaehler manifold of real dimension $2 n$ and $P$ is a connected compact real hypersurface of $M$.

The choice of $\mathscr{B}$ requires a bit of reflexion. We expect $\mathscr{V}$ to have a minimum on $\mathscr{B}$ and, if it is possible, to characterize the pairs on which this minimum is attained. In Heintze-Karcher's result, the set $\mathscr{R}_{n, \lambda, \Lambda}$ is determined by a bound $\lambda$ of a trace of the curvature operator of $M$ and a bound $\Lambda$ of the trace of the Weingarten map of $P$. Then the equality in (1.1) implies the equality of those traces with their bounds, and this makes the respective operators to be a multiple of the identity. Then the equality implies $M$ is of constant sectional curvature and $P$ is umbilical. Since there is no nonflat Kaehler manifold of constant sectional curvature, it is unlikely to find good results on sets determined by a bound of the Ricci curvature. On the other hand, it seems natural to expect the minima in some subsets of $\mathscr{K}_{n}$ to be among the nonflat Kaehler manifolds of constant holomorphic sectional curvature, but there is no umbilical real hypersurface in such Kaehler manifolds. All this forces us to choose carefully the invariants related to the curvature of $M$ and the Weingarten map of $P$ to be bounded to determine $\mathscr{B}$.

We adopt the following definition for the curvature and the tensor of Riemann-Christoffel:

$$
R(X, Y) Z=-\left[\nabla_{X}, \nabla_{Y}\right] Z+\nabla_{[X, Y]} Z
$$

and

$$
R_{X Y Z W}=\langle R(X, Y) Z, W\rangle \text {. }
$$

Let $(M ;\langle\rangle ; J$,$) be a Kaehler manifold. Recall that a holomorphic$ plane is that generated by two vectors of the form $X, J X$, and an antiholomorphic plane is that generated by two vectors, $X, Y$ such that $Y$ is orthogonal to both $X$ and $J X$.

1.1. Definition. The holomorphic (antiholomorphic) sectional curvature $K_{H}\left(K_{A}\right)$ of a $2 n$-dimensional Kaehler manifold $(M ;\langle\rangle ; J$, is the restriction of the sectional curvature of $M$ to the holomorphic (antiholomorphic) planes. The antiholomorphic Ricci curvature is the 
quadratic form $\rho_{A}$ defined on $T_{x} M$ for each $x \in M$ by

$$
\rho_{A}(X)=\sum_{k=1}^{2 n-2} R_{X e_{i} X e_{i}}, \quad \text { for every } X \in T_{x} M,
$$

where $\left\{e_{1}, \ldots, e_{2 n-2}, e_{2 n-1}, J e_{2 n-1}\right\}$ is an orthonormal basis of $T_{x} M$ such that $e_{2 n-1}=X /|X|$.

Observe that $\rho_{A}$ is a sum of antiholomorphic sectional curvatures. We also remark that $\rho(X, X)=\rho_{A}(X)+K_{H}(X)|X|^{2}$, where $\rho$ is the Ricci curvature of $M$ and $K_{H}(X)$ is the holomorphic sectional curvature of the plane generated by $X$ and $J X$.

Bounds on $K_{H}$ and $K_{A}$ have been used in [Gr2] for the related problem of getting comparison theorems for the volume of a tube about a complex submanifold of a Kaehler manifold.

1.2. Definition. Let $P$ be a real hypersurface of a Kaehler manifold $(M ;\langle\rangle ; J$,$) of real dimension 2 n$. Let $N$ be a unit vector field normal to $P$ defined on an open $U$ of $P$. Let $L$ be the Weingarten map of $P$ associated to $N$. We define the $J N$-normal curvature of $P$ at $p \in U, k_{J N}$, as the normal curvature of $P$ at $p$ in the direction $J N$ with the orientation given by $N$, i.e. $k_{J N}(p)=\langle L J N, J N\rangle(p)$. We define the $J N$-mean curvature of $P$ at $p$ as the real number

$$
H_{J N}(p)=\frac{1}{2 n-2}\left(\operatorname{tr} L-K_{J N}\right)(p)=\frac{(2 n-1) H-k_{J N}}{2 n-2}(p),
$$

where $H(p)$ is the mean curvature of $P$ at $p$.

From now on, unless otherwise stated, $M$ will denote a connected compact Kaehler manifold of real dimension $2 n$, with metric $\langle$,$\rangle and$ almost-complex structure $J . P$ will denote a connected compact real hypersurface of $M$.

We will denote by $\mathbb{C} P^{n}(\lambda)$ the complex projective space of real dimension $2 n$ and holomorphic (antiholomorphic) sectional curvature $4 \lambda(\lambda) . \quad S_{r}^{\complement}\left(B_{r}^{\complement}\right)$ will denote the geodesic sphere (ball) of radius $r$ in $\mathbb{C} P^{n}(\lambda) . T_{(\pi / 2 \sqrt{\lambda})-r}^{\mathbb{C}}=\mathbb{C} P^{n}(\lambda)-B_{r}^{\mathbb{C}}$ will denote the geodesic tube of radius $(\pi / 2 \sqrt{\lambda})-r$ about the complex submanifold $\mathbb{C} P^{n-1}(\lambda)$ of $\mathbb{C} P^{n}(\lambda)$.

The main results we shall prove here are

1.3. THEOREM. Let $\lambda, h, k$ be positive real numbers. Suppose that, on $M, K_{H} \geq 4 \lambda, \rho_{A} \geq(2 n-2) \lambda$. Suppose that, on $P, k_{J N} H_{J N} \geq 0$, 
$\left|H_{J N}\right| \leq h,\left|k_{J N}\right| \leq k$. Then

$$
\operatorname{vol}(M) \leq \frac{\operatorname{vol}\left(\mathbb{C} P^{n}(\lambda)\right)}{\operatorname{vol}\left(S_{r}^{\mathbb{C}}\right)} \operatorname{vol}(P),
$$

where $r=\min \left\{r_{k}, r_{h}\right\}$, and $r_{k}, r_{h}$ are defined by $k=2 \sqrt{\lambda} \cot \left(2 \sqrt{\lambda} r_{k}\right)$, and $h=\sqrt{\lambda} \cot \left(\sqrt{\lambda} r_{h}\right), 0 \leq \sqrt{\lambda} r_{h}, 2 \sqrt{\lambda} r_{k} \leq \pi / 2$. The equality holds if and only if $r_{k}=r_{h}$ and there is a holomorphic isometry $i: M \rightarrow \mathbb{C} P^{n}(\lambda)$ such that $i(P)=S_{r}^{\mathrm{C}}$.

Of course, when $n=1,(1.1)$ and (1.3.1) are equivalent.

Let $\mathscr{K}_{n, \lambda, h, k}$ be the family of pairs $(P, M)$ with $M$ and $P$ as in 1.3. Let $B(n, \lambda, h, k)=\left(\operatorname{vol}\left(S_{r}^{\mathbb{C}}\right)\right) /\left(\operatorname{vol}\left(\mathbb{C} P^{n}(\lambda)\right)\right)$. Denote $\mathscr{K}_{n, \lambda, h, k}$ by $\mathscr{K}_{n, \lambda, r}$ and $B(n, \lambda, h, k)$ by $B(n, \lambda, r)$ when $r_{k}=r_{h}=r$ (then $0 \leq r \leq \pi /(4 \sqrt{\lambda})$ ). Then 1.3 says: " $B(n, \lambda, h, k)$ is a lower bound of $\mathscr{V}$ restricted to $\mathscr{K}_{n, \lambda, h, k}$. This bound is a minimum only when $r_{k}=r_{h}=r$, and $\left(S_{r}^{\mathrm{C}}, \mathbb{C} P^{n}(\lambda)\right)$ is the only pair where this minimum is attained". Evidently $\mathscr{R}_{n, \lambda, r} \subset \mathscr{R}_{2 n, \lambda^{\prime}, \Lambda^{\prime}}$, where $\lambda^{\prime}=((2 n+2) /(2 n-1)) \lambda, \Lambda^{\prime}=$ $((2 n-2) h+k) /(2 n-1)$ and, from the Heintze-Karcher's result, $B(n, \lambda, r)>C\left(2 n, \lambda^{\prime}, \Lambda^{\prime}\right)$. By continuity it follows that, if $\left(r_{k}, r_{h}\right)$ is in a sufficiently small neighbourhood of $(r, r)$, then $B(n, \lambda, h, k)>$ $C\left(2 n, \lambda^{\prime}, \Lambda^{\prime}\right)$.

1.4. TheOREM. Assume $M$ satisfies the same hypotheses as in 1.3, and that $P$ is orientable. Let $h_{i}>0, k_{i}(i=1,2)$ be real numbers such that $k_{1} \leq k_{J N} \leq k_{2}, h_{1} \leq H_{J N} \leq h_{2}$ for a given orientation on $P$. Then

$$
\operatorname{vol}(M) \leq\left(\frac{\operatorname{vol}\left(B_{r_{1}}^{\mathrm{C}}\right)}{\operatorname{vol}\left(S_{r_{1}}^{\mathrm{C}}\right)}+\frac{\operatorname{vol}\left(T_{(\pi / 2 \sqrt{\lambda})-r_{2}}^{\mathrm{C}}\right)}{\operatorname{vol}\left(S_{r_{2}}^{\mathrm{C}}\right)}\right) \operatorname{vol}(P),
$$

where $r_{1}=\max \left\{r_{k_{1}}, r_{h_{1}}\right\}, r_{2}=\min \left\{r_{k_{2}}, r_{h_{2}}\right\}$ and $r_{k_{i}}, r_{h_{i}}$ are defined by $k_{i}=2 \sqrt{\lambda} \cot \left(2 \sqrt{\lambda} r_{k_{i}}\right)$ and $h_{i}=\sqrt{\lambda} \cot \left(\sqrt{\lambda} r_{h_{i}}\right), 0<r_{h_{i}}, r_{k_{i}}<\pi /(2 \sqrt{\lambda})$. The equality holds if and only if $r_{1}=r_{2}=r_{k_{i}}=r_{h_{i}} \equiv r$ and there is a holomorphic isometry $i: M \rightarrow \mathbb{C} P^{n}(\lambda)$ such that $i(P)=S_{r}^{\mathbb{C}}$.

Notice that if $\mathscr{H}$ is the harmonic mean of

$$
\frac{\operatorname{vol}\left(S_{r_{1}}^{\complement}\right)}{\operatorname{vol}\left(B_{r_{1}}^{\complement}\right)} \quad \text { and } \quad \frac{\operatorname{vol}\left(S_{r_{2}}^{\complement}\right)}{\operatorname{vol}\left(T_{(\pi / 2 \sqrt{\lambda})-r_{2}}^{\complement}\right)},
$$


i.e.

$$
\frac{2}{\mathscr{H}}=\frac{1}{\operatorname{vol}\left(S_{r_{1}}^{\mathbb{C}}\right) / \operatorname{vol}\left(B_{r_{1}}^{\mathbb{C}}\right)}+\frac{1}{\operatorname{vol}\left(S_{r_{2}}^{\mathbb{C}}\right) / \operatorname{vol}\left(T_{(n / 2 \sqrt{\lambda})-r_{2}}^{\mathrm{C}}\right)},
$$

then $(1.4 .1)$ can be written as $(\operatorname{vol}(P) / \operatorname{vol}(M)) \geq(\mathscr{H} / 2)$.

The hypothesis of the orientability of $P$ in (1.4.1) is of technical nature: it is necessary to fix the direction of $N$ at any point of $P$ in order to give universal bounds of $k_{J N}$ and $H_{J N}$.

Let $\mathscr{K}_{n, \lambda, h_{l}, k_{i}}$ be the family of pairs $(P, M)$ with $M$ and $P$ as in 1.4. Let $B\left(n, \lambda, h_{i}, k_{i}\right)=\mathscr{H} / 2$. Denote this $\mathscr{K}$ and $B$ by $\mathscr{K}_{n, \lambda, r}^{\prime}$ and $B^{\prime}(n, \lambda, r)$ when $r_{k_{l}}=r_{h_{l}}=r$ (then $0<r<(\pi /(2 \sqrt{\lambda})$ ). Then 1.4 says: " $\mathscr{V}$ restricted to $\mathscr{K}_{n, \lambda, h_{i}, k_{t}}$ has a lower bound $B\left(n, \lambda, h_{i}, k_{i}\right)$. This bound is a minimum only when $r_{k_{l}}=r_{h_{l}}=r$, and this minimum is attained only on the pair $\left(S_{r}^{\mathbb{C}}, \mathbb{C} P^{n}(\lambda)\right)$ ". For $0<r \leq \pi /(4 \sqrt{\lambda})$ we have $\mathscr{K}_{n, \lambda, r}^{\prime} \subset \mathscr{K}_{n, \lambda, r}$ and $B^{\prime}(n, \lambda, r)=B(n, \lambda, r)$. We recall that $\mathscr{K}_{n, \lambda, r}$ and $B(n, \lambda, r)$ are not defined for $\pi /(4 \sqrt{\lambda})<r<\pi /(2 \sqrt{\lambda})$. It is also obvious that $\mathscr{K}_{n, \lambda, h_{l}, k_{l}} \subset \mathscr{R}_{2 n, \lambda^{\prime}, \Lambda^{\prime}}$ with $\lambda^{\prime}=((2 n+2) /(2 n-1)) \lambda, \Lambda^{\prime}=$ $(1 /(2 n-1)) \max \left\{(2 n-2) h_{2}+k_{2},-(2 n-2) h_{1}-k_{1}\right\}$, and also, from Heintze-Karcher's result, $B^{\prime}(n, \lambda, r)>C\left(2 n, \lambda^{\prime}, \Lambda^{\prime}\right)$. By continuity it follows that, if $\left(r_{k_{1}}, r_{k_{2}}, r_{h_{1}}, r_{h_{2}}\right)$ is in a sufficiently small neighbourhood of $(r, r, r, r)$, then $B\left(n, \lambda, h_{i}, k_{i}\right)>C\left(2 n, \lambda^{\prime}, \Lambda^{\prime}\right)$.

We shall prove these theorems almost simultaneously in three steps. In the first one ( $\S 2)$, we shall obtain a comparison result for the volume element in Fermi coordinates around $P$, following the ideas in [Gr1]. In a second step ( $\S 3)$ we shall do the corresponding integrations to obtain (1.3.1) and (1.4.1). Then ( $\$ 4)$ we discuss the equalities.

Finally, in $\S 5$, we state two results on the relative volume of the boundary of a regular domain of a Kaehler manifold which are obtained in the same form as 1.3 and 1.4 and may be useful to get isoperimetric inequalities for Kaehler manifolds.

We wish to thank F. Carreras and O. Gil-Medrano for useful comments. We are specially indebted to A. Montesinos for his constructive criticisms on earlier versions of this paper.

\section{The estimates for the volume element.}

2.1. For every $p \in P$ and every unit vector $N \in T_{p} M$ orthogonal to $T_{p} P$, let $\gamma_{N}(s)$ be the geodesic such that $\gamma_{N}(0)=p, \gamma_{N}^{\prime}(0)=N$. Let 
$f(N)=\inf \left\{t>0 \mid \gamma_{N}(t)\right.$ is a focal point of $\left.P\right\}$. For every $\left.t \in\right] 0, f(N)[$, there is a neighbourhood $U$ of $\gamma_{N}(t)$ and a neighbourhood $V$ of $p$ in $P$ such that $P(t)=U \cap\{m \in M \mid d(m, V)=t\}$ is a real hypersurface of $M$. Let $S(t)$ be the Weingarten map of $P(t)$ associated to a unit normal vector field $N^{t}$ defined on $P(t)$ as an extension of $\gamma_{N}^{\prime}(t)$. Then $S(t)$ satisfies the differential equation (see [Gr1]):

$$
S^{\prime}(t)=S(t)^{2}+R(t)
$$

where $S^{\prime}(t)=\nabla_{\gamma_{N}^{\prime}(t)} S(t)$, and $R(t) U=R\left(N^{t} U\right) N^{t}$ for every $U \in$ $T_{m} P(t), m=\gamma_{N}(t), R$ being the curvature tensor of $M$.

Denote by $(\mathscr{S N} P) \mathscr{N} P$ the (unit) normal bundle of $P$ in $M$. Let $\omega$ be the riemannian volume element of $M$, and $d p$ that of $P$. Let $\theta_{N}(p, t)$ be the real function on $\{(p, N, t) \in \mathscr{S N} P \times \mathbf{R}: 0 \leq t<f(N)\}$ defined by $\omega\left(\gamma_{N}(t)\right)=\theta_{N}(p, t) d p \wedge d t$. Then $\theta_{N}$ satisfies the differential equation (see [Gr1]):

$$
\frac{\theta_{N}^{\prime}(p, t)}{\theta_{N}(p, t)}=-\operatorname{tr} S(t)
$$

2.2. Proposition. Suppose that, on $M, K_{H} \geq 4 \lambda, \rho_{A} \geq(2 n-2) \lambda$ $(\lambda>0)$. Denote by $\mathscr{N}_{p} P\left(\mathscr{S N}_{p} P\right)$ the fibre of $\mathscr{N} P(\mathscr{S N} P)$ at $p$. For each $p \in P$ and $N \in \mathscr{S N}_{p} P$, let $\left\{e_{i}\right\}_{1 \leq i \leq 2 n-1}$ be an orthonormal basis of $T_{p} P$ such that $e_{2 n-1}=J N$, and let $\left\{E_{i}(t)\right\}_{1 \leq i \leq 2 n-1}$ be parallel vector fields along $\gamma_{N}(t)$ such that $E_{i}(0)=e_{i}$ (this implies $\left.E_{2 n-1}(t)=J \gamma_{N}^{\prime}(t)\right)$. Then, if $L$ is the Weingarten map of $P$ at $p$ associated with the orientation given by $N$, and $L_{i j}=\left\langle L e_{i}, e_{j}\right\rangle$, we have

$$
\theta_{N}(p, t) \leq \mu_{N}(\lambda, p, t), \quad \text { where }
$$

$$
\begin{aligned}
\mu_{N}(\lambda, p, t)= & \left(\cos 2 \sqrt{\lambda} t-k_{J N}(p) \frac{\sin 2 \sqrt{\lambda} t}{2 \sqrt{\lambda}}\right) \\
& \times\left(\cos \sqrt{\lambda} t-H_{J N}(p) \frac{\sin \sqrt{\lambda} t}{\sqrt{\lambda}}\right)^{2 n-2}
\end{aligned}
$$

The equality in (2.2.1) is attained if and only if $L_{i i}=L_{j j}=\beta, 1 \leq$ $i, j \leq 2 n-2$, and with respect to $\left\{E_{i}(t)\right\}_{1 \leq i \leq 2 n-1}, S(t)$ and $R(t)$ have 
the matrix form

(2.2.3)

$$
\begin{aligned}
& S(t)=\left(\begin{array}{cccc}
-\frac{\delta_{\lambda}^{\prime}(\beta, t)}{\delta_{\lambda}(\beta, t)} & & 0 & \\
& \ddots & & \\
& & -\frac{\delta_{\lambda^{\prime}}(\beta, t)}{\delta_{\lambda}(\beta, t)} & \\
0 & & & -\frac{\zeta_{\lambda}^{\prime}\left(k_{J N}, t\right)}{\zeta_{\lambda}\left(k_{J N}, t\right)}
\end{array}\right), \\
& R(t)=\left(\begin{array}{llll}
\lambda & & 0 & \\
& \ddots & & \\
& & \lambda & \\
0 & & & 4 \lambda
\end{array}\right),
\end{aligned}
$$

where

$$
\begin{gathered}
\delta_{\lambda}(\beta, t)=\cos (\sqrt{\lambda} t)-\frac{\beta}{\sqrt{\lambda}} \sin (\sqrt{\lambda} t), \\
\zeta_{\lambda}\left(k_{J N}, t\right)=\cos (2 \sqrt{\lambda} t)-\frac{k_{J N}}{2 \sqrt{\lambda}} \sin (2 \sqrt{\lambda} t),
\end{gathered}
$$

and' denotes the derivative with respect to $t$.

Proof. Consider the functions

$$
f_{i}(t)=\left\langle S(t) E_{i}(t), E_{i}(t)\right\rangle
$$

Taking the derivative of both sides of (2.2.4), using (2.1.1) and the Cauchy-Schwarz inequality, we get

$$
\begin{aligned}
f_{i}^{\prime} & =\left\langle S^{\prime} E_{i}, E_{i}\right\rangle=\left\langle S^{2} E_{i}+R(t) E_{i}, E_{i}\right\rangle=\left\|S E_{i}\right\|^{2}+\left\langle R(t) E_{i}, E_{i}\right\rangle \\
& \geq\left\langle S E_{i}, E_{i}\right\rangle^{2}+\left\langle R(t) E_{i}, E_{i}\right\rangle=f_{i}^{2}+\left\langle R(t) E_{i}, E_{i}\right\rangle .
\end{aligned}
$$

But,

$$
\sum_{i=1}^{2 n-2}\left\langle R(t) E_{i}, E_{i}\right\rangle \geq(2 n-2) \lambda
$$

because, for $i=1, \ldots, 2 n-2$, the $E_{i}$ are perpendicular to both $\gamma_{N}^{\prime}$ and $J \gamma_{N}^{\prime}$, and $\rho_{A} \geq(2 n-2) \lambda$. We have also

$$
\left\langle R(t) E_{2 n-1}, E_{2 n-1}\right\rangle \geq 4 \lambda,
$$

since $K_{H} \geq 4 \lambda$. Then, we have the differential inequalities

$$
\begin{aligned}
\left(\frac{1}{2 n-2} \sum_{i=1}^{2 n-2} f_{i}\right)^{\prime} & \geq \frac{1}{2 n-2} \sum_{i=1}^{2 n-2} f_{i}^{2}+\lambda \\
& \geq\left(\frac{1}{2 n-2} \sum_{i=1}^{2 n-2} f_{i}\right)^{2}+\lambda
\end{aligned}
$$




$$
f_{2 n-1}^{\prime} \geq f_{2 n-1}^{2}+4 \lambda
$$

with

$$
f_{i}(0)=\left\langle L e_{i}, e_{i}\right\rangle=L_{i i}, \quad i=1, \ldots, 2 n-1 .
$$

Then ([Gr1, page 211]),

$$
\begin{gathered}
\frac{1}{2 n-2} \sum_{i=1}^{2 n-2} f_{i}(t) \geq \frac{\sqrt{\lambda} \sin \sqrt{\lambda} t+H_{J N} \cos \sqrt{\lambda} t}{\cos \sqrt{\lambda} t-\frac{H_{J N}}{\sqrt{\lambda}} \sin \sqrt{\lambda} t}, \\
f_{2 n-1}(t) \geq \frac{2 \sqrt{\lambda} \sin 2 \sqrt{\lambda} t+k_{J N} \cos 2 \sqrt{\lambda} t}{\cos 2 \sqrt{\lambda} t-\frac{k_{J N}}{2 \sqrt{\lambda}} \sin 2 \sqrt{\lambda} t},
\end{gathered}
$$

and the denominators of the right-hand sides of these inequalities are positive from $t=0$ to the first zero of each one.

Then, from (2.1.2),

$$
\text { (2.2.11) } \begin{aligned}
\frac{d}{d t} \ln \theta_{N}(p, t) & =-\operatorname{tr} S(t)=-\sum_{i=1}^{2 n-1}\left\langle S(t) E_{i}(t), E_{i}(t)\right\rangle \\
& \leq \frac{d}{d t} \ln \mu_{N}(\lambda, p, t) .
\end{aligned}
$$

Then $d\left(\ln \left(\theta_{N}(p, t) / \mu_{N}(\lambda, p, t)\right)\right) / d t \leq 0$, and $\theta_{N}(p, t) / \mu_{N}(\lambda, p, t)$ is a decreasing function of $t$, whose value for $t=0$ is 1 , whence (2.2.1) follows.

If we have the equality in (2.2.1), then all the inequalities in this proof must be equalities. Equality in (2.2.6) implies $f_{i}(t)=f_{j}(t) \equiv$ $\beta(t), 1 \leq i, j \leq 2 n-2$, and $\beta(0)=\beta \equiv L_{i i}=L_{j j}$. Equalities in (2.2.5) and (2.2.9) imply that $E_{i}(t)$ are eigenvectors of $S(t)$ with eigenvalue $-\delta_{\lambda}^{\prime}(\beta, t) / \delta_{\lambda}(\beta, t)$ for $1 \leq i \leq 2 n-2$ and $-\zeta_{\lambda}^{\prime}\left(k_{J N}, t\right) / \zeta_{\lambda}\left(k_{J N}, t\right)$ for $i=2 n-1$. This fact, (2.1.1) and the equalities in (2.2.6) and (2.2.7) give the matrix form of $R(t)$.

3. Proof of the inequalities (1.3.1) and (1.4.1).

3.1. Let $\mathbb{R}^{+}=\{t \in \mathbb{R}: t \geq 0\}, \mathbb{R}_{*}^{+}=\mathbb{R}^{+}-\{0\}$. For $i=1,2$ let $g_{i}: \mathbb{R}_{*}^{+} \times \mathbb{R}^{2} \times \mathbb{R}^{+} \rightarrow \mathbb{R}$ be the functions defined by

$$
g_{i}(\lambda, \alpha, \beta, t)=\zeta_{\lambda}\left(\varepsilon_{i} \alpha, t\right) \delta_{\lambda}\left(\varepsilon_{i} \beta, t\right)^{2 n-2},
$$

In general, given a function $q: X \times \mathbb{R}^{+} \rightarrow \mathbb{R}$, where $X$ is a given space, we denote by $z(q)$ the function which to every $x \in X$ associates the 
first zero of the function $t \rightarrow q(x, t)$. Then we define $g: \mathbb{R}_{*}^{+} \times \mathbb{R}^{2} \times \mathbb{R}^{2} \rightarrow$ $\mathbb{R}$ by

$$
g\left(\lambda, \alpha_{1}, \beta_{1}, \alpha_{2}, \beta_{2}\right)=\sum_{i=1}^{2} \int_{0}^{z\left(g_{i}\right)} g_{i}\left(\lambda, \alpha_{i}, \beta_{i}, t\right) d t,
$$

and $f: \mathbb{R}_{*}^{+} \times \mathbb{R}^{+} \times \mathbb{R}^{+} \rightarrow \mathbb{R}$ by

$$
f(\lambda, \alpha, \beta)=g(\lambda, \alpha, \beta, \alpha, \beta) .
$$

3.2. LEMMA. $f$ is an increasing function of $\alpha$ and $\beta$.

Proof. Since $\alpha, \beta>0$, we have

$$
z\left(g_{1}(\lambda, \alpha, \beta, t)\right) \leq \pi /(4 \sqrt{\lambda}) \leq z\left(g_{2}(\lambda, \alpha, \beta, t)\right) .
$$

Then from the definition of $f$ :

$$
\begin{aligned}
f(\lambda, \alpha, \beta)= & \int_{0}^{z\left(g_{1}\right)}\left(g_{1}+g_{2}\right)(\lambda, \alpha, \beta, t) d t \\
& +\int_{z\left(g_{1}\right)}^{z\left(g_{2}\right)} g_{2}(\lambda, \alpha, \beta, t) d t .
\end{aligned}
$$

Evidently $g_{2}$ is an increasing function of $\alpha$ and $\beta$. For $g_{1}+g_{2}$ we have, denoting

$$
\begin{aligned}
& a=\cos 2 \sqrt{\lambda} t, \quad b=(\sin 2 \sqrt{\lambda} t) /(2 \sqrt{\lambda}), \\
& \mathbf{a}=\cos \sqrt{\lambda} t \quad \text { and } \quad \mathbf{b}=(\sin \sqrt{\lambda} t) / \sqrt{\lambda},
\end{aligned}
$$

that

$$
\begin{gathered}
\left(g_{1}+g_{2}\right)(\lambda, \alpha, \beta, t)=(a-\alpha b)(\mathbf{a}-\beta \mathbf{b})^{2 n-2}+(a+\alpha b)(\mathbf{a}+\beta \mathbf{b})^{2 n-2} \\
=2 a \sum_{j=1}^{n-1}\left(\begin{array}{c}
2 n-2 \\
2 j
\end{array}\right) \mathbf{a}^{2 n-2-2 j} \mathbf{b}^{2 j} \beta^{2 j} \\
\quad+2 b \mathbf{a b} \alpha \sum_{j=1}^{n-1}\left(\begin{array}{c}
2 n-2 \\
2 j-1
\end{array}\right) \mathbf{a}^{2 n-2-2 j} \mathbf{b}^{2 j-2} \beta^{2 j-1}, \\
a=\cos 2 \sqrt{\lambda} t>0, \quad \text { for } t<\frac{\pi}{4 \sqrt{\lambda}}, \\
b \mathbf{a b}=\frac{\sin ^{2} 2 \sqrt{\lambda} t}{4 \lambda} \geq 0 .
\end{gathered}
$$


Then $g_{1}+g_{2}$ is increasing in $\alpha$ and $\beta$ for $0 \leq t<z\left(g_{1}\right)$. Then $\partial\left(g_{1}+g_{2}\right) / \partial \alpha>0$ and $\partial\left(g_{1}+g_{2}\right) / \partial \beta>0$, which implies

$$
\begin{aligned}
\frac{\partial}{\partial \alpha} f(\lambda, \alpha, \beta) & =\frac{\partial}{\partial \alpha} \int_{0}^{z\left(g_{1}\right)} g_{1} d t+\frac{\partial}{\partial \alpha} \int_{0}^{z\left(g_{2}\right)} g_{2} d t \\
& =\int_{0}^{z\left(g_{1}\right)} \frac{\partial}{\partial \alpha}\left(g_{1}+g_{2}\right) d t+\int_{z\left(g_{1}\right)}^{z\left(g_{2}\right)} \frac{\partial}{\partial \alpha} g_{2} d t>0,
\end{aligned}
$$

and, from a similar computation, $\frac{\partial}{\partial \beta} f(\lambda, \alpha, \beta)>0$. Then $f$ is an increasing function of $\alpha$ and $\beta$.

3.3. Proposition. (a) If $M$ and $P$ satisfy the same conditions as in 1.3 with the same bounds for $K_{H}, \rho_{A},\left|H_{J N}\right|$ and $\left|k_{J N}\right|$, then

(3.3.1) $\operatorname{vol}(M) \leq \int_{P} f\left(\lambda,\left|k_{J N}(p)\right|,\left|H_{J N}(p)\right|\right) d p \leq f(\lambda, k, h) \operatorname{vol}(P)$.

(b) If $M$ and $P$ satisfy the same conditions as in 1.4 with the same bounds for $K_{H}, \rho_{A}, H_{J N}$ and $k_{J N}$, then

$$
\text { (3.3.2) } \begin{aligned}
\operatorname{vol}(M) & \leq \int_{P} g\left(\lambda, k_{J N}(p), H_{J N}(p), k_{J N}(p), H_{J N}(p)\right) d p \\
& \leq g\left(\lambda, k_{1}, h_{1}, k_{2}, h_{2}\right) \operatorname{vol}(P) .
\end{aligned}
$$

Proof. Let $c(N)=\sup \left\{t>0: d\left(P, \gamma_{N}(t)\right)=t\right\}$, cut $P=\left\{\gamma_{N}(c(N))\right.$ : $N \in \mathscr{S N} P\}$. From the facts that

$$
M=\left\{\gamma_{N}(t): N \in \mathscr{S N} P, 0 \leq t<c(N)\right\} \cup \operatorname{cut} P,
$$

$\gamma_{N}$ is the only minimizing geodesic from $P$ to $\gamma_{N}(t)$ for all $\left.t \in\right] 0, c(N)[$, $c(N) \leq f(N)=z\left(\theta_{N}(p, t)\right)$, and the inequality (2.2.1), we have

$$
\begin{aligned}
\operatorname{vol}(M) & =\int_{P}\left\{\int_{0}^{c(N)} \theta_{N}(p, t) d t+\int_{0}^{c(-N)} \theta_{-N}(p, t) d t\right\} d p \\
& \leq \int_{P}\left\{\int_{0}^{z\left(\theta_{N}(p, t)\right)} \theta_{N}(p, t) d t+\int_{0}^{z\left(\theta_{-N}(p, t)\right)} \theta_{-N}(p, t) d t\right\} d p \\
& \leq \int_{P}\left\{\int_{0}^{z\left(\mu_{N}(\lambda, p, t)\right)} \mu_{N}(\lambda, p, t) d t\right. \\
& \left.+\int_{0}^{z\left(\mu_{-N}(\lambda, p, t)\right)} \mu_{-N}(\lambda, p, t) d t\right\} d p
\end{aligned}
$$

and the first part of inequalities (3.3.1) and (3.3.2) follow from this one, if we have in mind that the Weingarten maps of $P$ associated to 
$N$ and $-N$ have opposite sign and, then, $k_{J N}=-k_{J(-N)}$ and $H_{J N}=$ $-H_{J(-N)}$.

The second part of the inequality (3.3.2) follows immediately from the first, and the second of (3.3.1) follow from the first and Lemma 3.2 .

In order to prove (1.3.1) and (1.4.1) we need

3.4. LEMMA. Let $S_{r}^{\mathbb{C}}$ be the geodesic sphere of radius $r$ in $\mathbb{C} P^{n}(\lambda), p \in$ $S_{r}^{\mathbb{C}}$ and $N \in \mathscr{S N}_{p} S_{r}^{\mathbb{C}}$ pointing toward the centre of $S_{r}^{\mathbb{C}}$. Then there is an orthonormal basis of $T_{p} S_{r}^{\mathbb{C}}$ of the form $\left\{e_{i}\right\}_{1 \leq i \leq 2 n-1}$ such that $e_{2 n-1}=J N$ in which the Weingarten map of $S_{r}^{C}$ associated to $N$ in $\mathbb{C} P^{n}(\lambda)$ has the matrix form

$$
\left(\begin{array}{cccc}
\sqrt{\lambda} \cot \sqrt{\lambda} r & & 0 & \\
& \ddots & & \\
& & \sqrt{\lambda} \cot \sqrt{\lambda} r & \\
0 & & & 2 \sqrt{\lambda} \cot 2 \sqrt{\lambda} r
\end{array}\right) .
$$

Proof. An explicit expression for $\lambda=1$ is given in [C-R]. It can also be obtained using the methods of [C-V].

3.5. Proof of (1.3.1). Let $M=\mathbb{C} P^{n}(\lambda), P=S_{r}^{\mathbb{C}}$ in 2.2. If we take $\left\{e_{i}\right\}_{1 \leq i \leq 2 n-1}$ as the basis given in 3.4 , then all the inequalities of the proof are equalities and, then

$$
\theta_{N}^{C P^{n}(\lambda)}(p, t)=\mu_{N}(\lambda, p, t) .
$$

Moreover $c(N)=z\left(\mu_{N}(\lambda, p, t)\right)$, whence

$$
\operatorname{vol}\left(\mathbb{C} P^{n}(\lambda)\right)=\int_{S_{r}^{\mathrm{C}}} f(\lambda, 2 \sqrt{\lambda} \cot 2 \sqrt{\lambda} r, \sqrt{\lambda} \cot \sqrt{\lambda} r) d u,
$$

where $d u$ is the volume element of $S_{r}^{\mathbb{C}}$. Then

$$
\operatorname{vol}\left(\mathbb{C} P^{n}(\lambda)\right)=\operatorname{vol}\left(S_{r}^{\mathbb{C}}\right) f(\lambda, 2 \sqrt{\lambda} \cot 2 \sqrt{\lambda} r, \sqrt{\lambda} \cot \sqrt{\lambda} r) .
$$

If $r, r_{k}, r_{h}$ have the values given in 1.3 , then $2 \sqrt{\lambda} \cot 2 \sqrt{\lambda} r \geq k$ and $\sqrt{\lambda} \cot \sqrt{\lambda} r \geq h$, and, since $f$ is increasing in the last two arguments, we get (1.3.1) from (3.3.1) and (3.5.1).

3.6. Proof of (1.4.1). First observe that for $M=\mathbb{C} P^{n}(\lambda), P=S_{r}^{\mathbb{C}}$,

$$
\mu_{\varepsilon_{i} N}(\lambda, p, t)=g_{i}(\lambda, 2 \sqrt{\lambda} \cot 2 \sqrt{\lambda} r, \sqrt{\lambda} \cot \sqrt{\lambda} r, t) .
$$


Then, if $r_{i}, r_{k_{i}}, r_{h_{i}}$ are the quantities defined in 1.4, $\operatorname{vol}\left(B_{r_{1}}^{\mathrm{C}}\right)=\int_{S_{r_{1}}^{\mathrm{c}}} \int_{0}^{r_{1}} g_{1}\left(\lambda, 2 \sqrt{\lambda} \cot 2 \sqrt{\lambda} r_{1}, \sqrt{\lambda} \cot \sqrt{\lambda} r_{1}, t\right) d t d u$,

(3.6.2) $\operatorname{vol}\left(T_{(\pi / 2 \sqrt{\lambda})-r_{2}}^{\mathrm{C}}\right)$

$$
=\int_{S_{r_{2}}^{\mathrm{c}}} \int_{0}^{(\pi / 2 \sqrt{\lambda})-r_{2}} g_{2}\left(\lambda, 2 \sqrt{\lambda} \cot 2 \sqrt{\lambda} r_{2}, \sqrt{\lambda} \cot \sqrt{\lambda} r_{2}, t\right) d t d u .
$$

From the definition of the $r_{i}$ and $g_{i}$ :

$$
g_{i}\left(\lambda, 2 \sqrt{\lambda} \cot 2 \sqrt{\lambda} r_{i}, \sqrt{\lambda} \cot \sqrt{\lambda} r_{i}, t\right) \geq g_{i}\left(\lambda, k_{i}, h_{i}, t\right) .
$$

Then we get (1.4.1) from (3.6.1,2,3) and (3.3.2).

4. Equality discussion. First we recall some known facts about Jacobi operators. We take them from [Ch].

4.1. Definition. Let $p \in P, N \in \mathscr{S N}_{p} P, \gamma_{N}(t)$ as in 2.1. Let $\tau_{t}$ be the parallel transport along $\gamma_{N}(t)$. Then the Jacobi operator $A(t, N):\left\{\gamma_{N}^{\prime}(0)\right\}^{\perp} \rightarrow\left\{\gamma_{N}^{\prime}(0)\right\}^{\perp}$ is defined by

$$
A(t, N) e=\tau_{t}^{-1} Y(t),
$$

where $Y(t)$ is the transverse Jacobi field along $\gamma_{N}$ such that $Y(0)=e$, $\nabla_{\gamma_{N}^{\prime}(0)} Y \equiv Y^{\prime}(0)=-L e$.

4.2. Proposition [Ch]. $A(t, N)$ satisfies the differential equation:

$$
A^{\prime \prime}(t, N)+\mathscr{R}(t) A(t, N)=0
$$

with the initial conditions $A(0, N)=I, A^{\prime}(0, N)=-L$, where $\mathscr{R}(t)=$ $\tau_{t}^{-1} R(t) \tau_{t}$.

Observe that if $\left\{E_{i}\right\}$ is a basis of $\left\{\gamma_{N}^{\prime}(t)\right\}^{\perp}$ obtained by parallel transport of a basis $\left\{e_{i}\right\}$ of $\left\{\gamma_{N}^{\prime}(0)\right\}^{\perp}$, then the matrix of $R(t)$ in the basis $\left\{E_{i}\right\}$ and that of $\mathscr{R}(t)$ in the basis $\left\{e_{i}\right\}$ are the same.

4.3. Remark. For every subset $\mathbf{S}$ of $T M$, denote by $\exp _{\mathbf{S}}$ the restriction to $\mathbf{S}$ of the exponential map on $T M$. Let $\mathscr{N} P(t)=\{X \in$ $\mathscr{N} P$ such that $|X|=t\}$. From Definition 4.1 it is obvious that

$$
\operatorname{rank}(A(t, N))=\operatorname{rank}\left(\exp _{\mathscr{N} P(t)^{*} t N}\right) \text {. }
$$

4.4. Definition. Let $m \in M, \gamma_{\xi}(t)$ a geodesic parametrized by its arc length starting from $m\left(\gamma_{\xi}(0)=m\right)$, with $\gamma_{\xi}^{\prime}(0)=\xi$. Then the Jacobi operator $A_{m}(t, \xi):\{\xi\}^{\perp} \rightarrow\{\xi\}^{\perp}$ is defined by

$$
A_{m}(t, \xi) e=\tau_{t}^{-1} Y(t),
$$

where $Y(t)$ is the jacobi field along $\gamma_{\xi}(t)$ such that $Y(0)=0, Y^{\prime}(0)=e$. 
4.5. Proposition [Ch]. $A_{m}(t, \xi)$ satisfies the differential equation

$$
A_{m}^{\prime \prime}(t, \xi)+\mathscr{R}_{m}(t) A_{m}(t, \xi)=0
$$

with the initial conditions $A_{m}(0, \xi)=0, A_{m}^{\prime}(0, \xi)=I$, where $\mathscr{R}_{m}(t)$ : $\{\xi\}^{\perp} \rightarrow\{\xi\}^{\perp}$ is defined by

$$
\mathscr{R}_{m}(t) e=\tau_{t}^{-1} R\left(\gamma_{\xi}^{\prime}(t), \tau_{t} e\right) \gamma_{\xi}^{\prime}(t)
$$

4.6. Proposition [Ch]. Let $\xi^{-1}$ be a coordinate system of the euclidean sphere $S^{2 n-1}$ of $T_{m} M$. Then $x(t, u)=\exp _{m} t \xi(u)$ defines $a$ system of polar geodesic coordinates $x^{-1}$ around $m$. In this coordinate system the metric tensor has the expression

$$
d s^{2}=d t^{2}+\sum_{i, j=1}^{2 n-1}\left\langle A_{m}(t, \xi(u)) \frac{\partial \xi}{\partial u^{i}}(u), A_{m}(t, \xi(u)) \frac{\partial \xi}{\partial u^{j}}(u)\right\rangle d u^{i} d u^{j}
$$

4.7. Proposition [Ch]. Let $\varphi^{-1}$ be a coordinate system of $P \subset M$. Then $v(t, u)=\exp _{\varphi(u)} t N(u)$ defines a system of Fermi coordinates $v^{-1}$ around $P$. In this coordinate system the metric tensor has the expression $d s^{2}=d t^{2}+\sum_{i, j=1}^{2 n-1}\left\langle A(t, N(\varphi(u))) \frac{\partial \varphi(u)}{\partial u^{i}}, A(t, N(\varphi(u))) \frac{\partial \varphi(u)}{\partial u^{j}}\right\rangle d u^{i} d u^{j}$

In order to prove 1.3 and 1.4 it only remains to know what happens when equality occurs in (1.3.1) or (1.4.1). To do it we observe the following facts:

4.8. To prove (1.3.1) we used in 3.5 that

$$
f(\lambda, k, h) \leq f(\lambda, 2 \sqrt{\lambda} \cot 2 \sqrt{\lambda} r, \sqrt{\lambda} \cot \sqrt{\lambda} r)
$$

and equality implies $r=r_{k}=r_{h}$ (i.e. $k=2 \sqrt{\lambda} \cot 2 \sqrt{\lambda} r$ and $h=$ $\sqrt{\lambda} \cot \sqrt{\lambda} r$.

4.9. Equality in (1.3.1) implies equality in (3.3.1) and then, looking at the proof of (3.3.1) we observe that equality implies $c(N)=f(N)=$ $z\left(\theta_{N}(\lambda, p, t)\right)=z\left(\mu_{N}(\lambda, p, t)\right)=r$.

4.10. Equality in (3.3.1) implies $\left|k_{J N}(p)\right|=k,\left|H_{J N}(p)\right|=h$, because $f$ is an increasing function. Then, for every $p \in P$ we can take $N \in \mathscr{S} \mathscr{N}_{P} P$ such that, for the Weingarten map $L$ of $P$ associated to $N, k_{J N}=k$ and $H_{J N}=h$. In particular, $P$ is orientable, $\mathscr{S N} P$ has two connected components and the subset $\mathscr{A}$ of such $N$ is one of them. With this choice, equality in (1.3.1) implies equality in 
(2.2.1) and, from 2.2, with respect to the basis given in 2.2, $L$ has a diagonal matrix with $L_{i i}=h=\sqrt{\lambda} \cot \sqrt{\lambda} r$ for $1 \leq i \leq 2 n-2$ and $L_{2 n-12 n-1}=k=2 \sqrt{\lambda} \cot 2 \sqrt{\lambda} r$, and $\mathscr{R}(t)$ has the matrix expression given in (2.2.3).

4.11. Let $\mathscr{A}$ be the set of the $N \in \mathscr{S N} P$ considered in 4.10. Then, from 4.2 and 4.10 we have that equality in (1.3.1) implies

$$
A(t, \pm N)=\left(\begin{array}{ccc}
\delta_{\lambda}( \pm \sqrt{\lambda} \cot \sqrt{\lambda} r, t) & & 0 \\
0 & \dot{\delta}_{\lambda}( \pm \sqrt{\lambda} \cot \sqrt{\lambda} r, t) & 0 \\
& & \zeta_{\lambda}( \pm 2 \sqrt{\lambda} \cot 2 \sqrt{\lambda} r, t)
\end{array}\right)
$$

The matrices $A(t, N), N \in \mathscr{A}$, have rank $2 n-1$ for $0 \leq t<r$, and rank 0 for $t=r$. Then, from $4.3, \operatorname{rank}\left(\exp _{\mathscr{N} P(r)^{*} r N}\right)=0$ for every $N \in \mathscr{A}$. This implies that there is a point $m \in M$ such that $\exp _{\mathscr{N} P}(\{r N, N \in \mathscr{A}\})=\{m\}$.

In the following assertions we always assume the equality in (1.3.1) (and hence in (3.3.1)).

4.12. For $N \in \mathscr{A}$, equality in (3.3.1) implies (as in 4.9) $c(-N)=$ $f(-N)=z\left(\mu_{-N}(\lambda, p, t)\right)=(\pi / 2 \sqrt{\lambda})-r$. Then the focal set and the cutfocal points of $P$ in the direction of $-N$ are focal $-(P)=$ cut $_{-}(P)=$ $\left\{\gamma_{-N}((\pi / 2 \sqrt{\lambda})-r): N \in \mathscr{A}\right\}$.

4.13. As a consequence of 4.11 every point $p \in P$ can be joined to $m$ by a geodesic $\gamma_{N}(t), N \in \mathscr{A}$, such that $\gamma_{N}(r)=m, m$ is the first focal point of $P$ in the direction $N$ and $r=c(N)$. Let $N(p)$ be the unique element of $\mathscr{A} \cap T_{p} M$. Let $S^{2 n-1}$ be the unit sphere in $T_{m} M$. Let us consider the continuous map $\Phi: P \rightarrow S^{2 n-1}$ given by $\Phi(p)=-\gamma_{N(p)}^{\prime}(r)=-\left(d\left(\exp _{\mathscr{N} P} t N(p)\right) / d t\right)(r)$. Since $P$ is compact, we have that $\Phi(P)$ is closed in $S^{2 n-1}$.

Let $\varepsilon \in \mathbb{R}, 0<\varepsilon<(\pi / 2 \sqrt{\lambda})-r$ and $V=\exp _{\mathscr{N} P}\{t N:-\varepsilon<t<r$, $N \in \mathscr{A}\}=\exp _{m}\{s \xi: 0<s<r+\varepsilon, \xi \in \Phi(P)\}$. Then it follows from 4.11 and 4.12 that $V$ is open. Let $z: \mathbb{R}_{*}^{+} \times S^{2 n-1} \rightarrow T_{m} M-\{0\}$ be the diffeomorphism given by $z(s, \xi)=s \xi$. Then $] 0, r+\varepsilon[\times \phi(P)=$ $z^{-1} \exp _{m}^{-1}(V)$ is open, then $\Phi(P)$ is open in $S^{2 n-1}$. Since $S^{2 n-1}$ is connected, we have that $\Phi(P)=S^{2 n-1}$ and, then, $P=S_{m}(r)$, the geodesic sphere of $M$ of center $m$ and radius $r$. 
4.14. From 4.5 and (2.2.3) we have, for every $\xi \in S^{2 n-1} \subset T_{m} M$,

$$
A_{m}(s, \xi)=\left(\begin{array}{cccc}
\frac{1}{\sqrt{\lambda}} \sin \sqrt{\lambda} s & & & \\
& \ddots & & 0 \\
0 & & \frac{1}{\sqrt{\lambda}} \sin \sqrt{\lambda} s & \\
& & & \frac{1}{2 \sqrt{\lambda}} \sin 2 \sqrt{\lambda} s
\end{array}\right)
$$

Then

$$
\operatorname{rank} A_{m}(s, \xi)=2 n-1 \quad \text { for } 0<s<\pi /(2 \sqrt{\lambda})
$$

and

$$
\operatorname{rank} A_{m}(\pi /(2 \sqrt{\lambda}), \xi)=2 n-2
$$

which implies that $c_{0}(\xi):=\min \left\{t>0: \gamma_{\xi}(t)\right.$ is a conjugate point of $m$ along $\left.\gamma_{\xi}\right\}=\pi /(2 \sqrt{\lambda})$. Then, the set of conjugated points of $m$ is $\operatorname{conj}(m)=\left\{\gamma_{\xi}(\pi /(2 \sqrt{\lambda})), \xi \in S^{2 n-1} \subset T_{m} M\right\}$ and, given $\xi \in S^{2 n-1} \subset$ $T_{m} M$, if $N \in \mathscr{A}$ is such that $\xi=-\gamma_{N}^{\prime}(r)$, then $\gamma_{\xi}(s)=\gamma_{N}(r-s)=$ $\gamma_{-N}(s-r)$.

4.15. From 4.14, $\gamma_{\xi}(\pi /(2 \sqrt{\lambda}))=\gamma_{-N}(\pi /(2 \sqrt{\lambda})-r)$. Then from 4.12 and $4.14 \operatorname{conj}(m)=$ cut $_{-}(P)=\operatorname{cut}(m)$, where cut $(m)$ is the cut locus of $m$. This allows us to define the map $i^{\prime}$

$$
i^{\prime}: M-\operatorname{cut}(m) \rightarrow \mathbb{C} P^{n}(\lambda)-\operatorname{cut}\left(m^{\prime}\right)
$$

in the following form: Let $j: T_{m} M \rightarrow T_{m^{\prime}} \subset P^{n}(\lambda)$ be a holomorphic isometry, then we define

$$
i^{\prime}\left(\exp _{m} s \xi\right)=\exp _{m^{\prime}} s j(\xi), \quad \text { for } 0 \leq s<\pi / 2 \sqrt{\lambda}, \quad \xi \in S^{2 n-1} .
$$

Since $\mathscr{R}_{m}(t)$ is the same map for $M$ and for $\mathbb{C} P^{n}(\lambda)$, we have $A_{m}(s, \xi)=A_{m^{\prime}}(s, j(\xi))$. If $\xi^{-1}$ is a coordinate system in $S^{2 n-1} \subset T_{m} M$ which defines polar geodesic coordinates $x^{-1}$ around $m$ as in 4.6, $\xi^{\prime-1}=(j \circ \xi)^{-1}$ defines polar geodesic coordinates $x^{\prime-1}$ around $m^{\prime}$ such that $i^{\prime} \circ x^{-1}=x^{\prime-1}$. Then, from 4.6, $i^{\prime}$ is a holomorphic isometry.

4.16. Since the map $i^{\prime}$ in (4.15.1) is a holomorphic isometry, $M-$ $\operatorname{cut}(m)$ has constant holomorphic sectional curvature. Moreover, $M-$ $\operatorname{cut}(m)$ is dense in $M$, whence by continuity, $M$ has constant holomorphic sectional curvature, and, $M$ being compact, there is a holomorphic isometry $i: M \rightarrow \mathbb{C} P^{n}(\lambda)$ (cf. [K-N]). Let $m^{\prime}=i(m)$ and $j=i *$. Then $i_{\mid M-\operatorname{cut}(m)}=i^{\prime}$, which implies (by 4.13) that $i(P)=i^{\prime}\left(S_{m}(r)\right)=$ $S_{r}^{\mathbb{C}}$. This finishes the proof of Theorem 1.3. 
To prove 1.4, observe that equality in (1.4.1) implies that (3.6.3) are equalities, and, then, $r_{i}=r_{k_{i}}=r_{h_{i}}$. Also, equality in (1.4.1) implies equalities in (3.3.2) and, then $k_{i}=k_{J N}, h_{i}=H_{J N}$, and $r_{i}=r$. Then, $H_{J N}=\sqrt{\lambda} \cot (\sqrt{\lambda} r), k_{J N}=2 \sqrt{\lambda} \cot (2 \sqrt{\lambda} r)$, and, from here, the proof of 1.4 follows from similar arguments to those used in 1.3.

5. Inequalities on the relative volume of the boundary of a domain in a Kaehler manifold. The proof of 1.4 also proves, with slight modifications, the following result:

5.1. THEOREM. Let $M$ be as in 1.3. Let $\Omega$ be a compact regular domain of $M$ with boundary $\partial \Omega=P$. For every $p \in P$ take $N \in \mathscr{S N}_{p} P$ such that $N$ points toward $\Omega$ (i.e. $\gamma_{N}(t) \in \Omega$ for small $t>0$ ).

(a) Let $h_{1}>0, k_{1}$ be real numbers such that $k_{1} \leq k_{J N}(p), h_{1} \leq$ $H_{J N}(p)$ for every $p \in P$. Then:

$$
\frac{\operatorname{vol}(\partial \Omega)}{\operatorname{vol}(\Omega)} \geq \frac{\operatorname{vol}\left(S_{r_{1}}^{C}\right)}{\operatorname{vol}\left(B_{r_{1}}^{C}\right)}
$$

where $r_{k_{1}}, r_{h_{1}}, r_{1}$ are defined as in 1.4. When $\Omega$ and $\partial \Omega$ are connected, the equality holds if and only if $r_{1}=r_{h_{1}}=r_{k_{1}}$ and there is a holomorphic isometry $i: \Omega \rightarrow B_{r}^{\mathrm{C}}$ such that $i(\partial \Omega)=S_{r}^{\mathrm{C}}$.

(b) Let $h_{2}, k_{2}$ be real numbers such that $k_{J N}(p) \leq k_{2}, H_{J N}(p) \leq h_{2}$. Then

$$
\frac{\operatorname{vol}(\partial \Omega)}{\operatorname{vol}(M-\Omega)} \geq \frac{\operatorname{vol}\left(S_{r_{2}}^{\mathbb{C}}\right)}{\operatorname{vol}\left(T_{\left.(\pi / 2 \sqrt{\lambda})-r_{2}\right)}^{\mathbb{C}}\right.},
$$

where $r_{2}=\min \left\{r_{h_{2}}, r_{k_{2}}\right\}, r_{k_{2}}$ is defined as in 1.4, and $r_{h_{2}}$ is defined by $h_{2}=\sqrt{\lambda} \cot \sqrt{\lambda} r_{h_{2}}, 0<r_{h_{2}}<\pi / \sqrt{\lambda}$. The equality in (5.1.2) implies $r_{h_{2}}=r_{k_{2}}=r_{2}$.

5.2. Corollary. Let $M, \Omega, \partial \Omega$ and $N$ be as in 5.1 (a), but now $h_{1} \leq 0$. Then

$$
\frac{\operatorname{vol}(\partial \Omega)}{\operatorname{vol}(\Omega)} \geq \frac{\operatorname{vol}\left(S_{r_{1}}^{\mathbb{C}}\right)}{\operatorname{vol}\left(T_{(\pi / 2 \sqrt{\lambda})-r_{1}}^{\mathbb{C}}\right)},
$$

where $r_{1}=\min \left\{r_{k_{1}}, r_{h_{1}}\right\}$, and $r_{k_{1}}, r_{h_{1}}$ are defined by $-h_{1}=\sqrt{\lambda} \cot \sqrt{\lambda} r_{h_{1}}$, $-k_{1}=2 \sqrt{\lambda} \cot 2 \sqrt{\lambda} r_{k_{1}}, 0<r_{h_{1}}, r_{k_{1}}<\pi / 2 \sqrt{\lambda}$. The equality in (5.2.1) implies $r_{k_{1}}=r_{h_{1}}$.

Proof. $M-\Omega, \partial \Omega$ and $-N$ satisfy the condition of 5.1 (b) with $h_{2}=-h_{1}$ and $k_{2}=-k_{1}$. Then the $r_{h_{2}}$ defined in 5.1(b) is less than 
or equal to $\pi / 2 \sqrt{\lambda}$, because $h_{2}=-h_{1} \geq 0$. Then the result of $5.1(\mathrm{~b})$ holds and we get 5.2.

\section{REFERENCES}

[Ch] I. Chavel, Eigenvalues in Riemannian Geometry, Academic Press 1984.

[C-R] T. E. Cecil and P. J. Ryan, Focal sets and real hypersurfaces in complex projective space, Trans. Amer. Math. Soc. 269 (1982), 481-499.

[C-V] B. Y. Chen and L. Vanhecke, Differential geometry of geodesic spheres, J. Reine Angew. Math. 325 (1981), 28-67.

[Gr1] A. Gray, Comparison theorems for the volumes of tubes as generalizations of the Weyl tube formula, Topology, 21 (1982), 201-228.

[Gr2] A. Gray, Volumes of tubes about complex submanifolds of complex projective space, Trans. Amer. Math. Soc., 291 (1985), 437-449.

[H-K] E. Heintze and H. Karcher, A general comparison theorem with applications to volume estimates for submanifolds, Ann. Sci. Ec. Norm. Sup. 11 (1978), 451-470.

[K-N] S. Kobayashi and K. Nomizu, Foundations of Differential Geometry, Vol II, Interscience 1969.

Received March 1, 1988 and in revised form January 11, 1989. Work partially supported by a DGICYT Grant No. PS87-0115-C03-01.

Departamento de Geometria y Topologia

UNIVERSIDAD DE VALENCIA

BurJassot (VAlencia) Spain 



\section{PACIFIC JOURNAL OF MATHEMATICS EDITORS}

\author{
V. S. VARADARAJAN \\ (Managing Editor) \\ University of California \\ Los Angeles, CA 90024-1555-05 \\ Herbert Clemens \\ University of Utah \\ Salt Lake City, UT 84112 \\ ThOMAS ENRIGHT \\ University of California, San Diego \\ La Jolla, CA 92093
}

\section{R. FINN}

Stanford University

Stanford, CA 94305

HeRmann FlaschKa

University of Arizona

Tucson, AZ 85721

VAUGHAN F. R. JONES

University of California

Berkeley, CA 94720

Steven KerckhofF

Stanford University

Stanford, CA 94305

\author{
RobION KIRBY \\ University of California \\ Berkeley, CA 94720 \\ C. C. Moore \\ University of California \\ Berkeley, CA 94720 \\ HAROLD STARK \\ University of California, San Diego \\ La Jolla, CA 92093
}

\section{ASSOCIATE EDITORS}
R. ARENS
E. F. BECKenBach
B. H. NeumanN
F. WOLF
K. YoshidA (1906-1982)
(1904-1989)

\section{SUPPORTING INSTITUTIONS}

UNIVERSITY OF ARIZONA

UNIVERSITY OF BRITISH COLUMBIA

CALIFORNIA INSTITUTE OF TECHNOLOGY

UNIVERSITY OF CALIFORNIA

MONTANA STATE UNIVERSITY

UNIVERSITY OF NEVADA, RENO

NEW MEXICO STATE UNIVERSITY

OREGON STATE UNIVERSITY
UNIVERSITY OF OREGON

UNIVERSITY OF SOUTHERN CALIFORNIA

STANFORD UNIVERSITY

UNIVERSITY OF HAWAII

UNIVERSITY OF TOKYO

UNIVERSITY OF UTAH

WASHINGTON STATE UNIVERSITY

UNIVERSITY OF WASHINGTON 


\section{Pacific Journal of Mathematics}

Vol. 142, No. $1 \quad$ January, 1990

Marco Andreatta, Mauro Beltrametti and Andrew Sommese, Generic properties of the adjuction mapping for singular surfaces and applications

Chen-Lian Chuang and Pjek-Hwee Lee, On regular subdirect products of

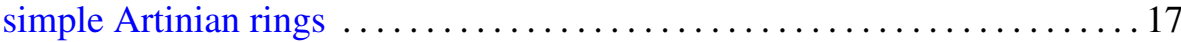

Fernando Giménez and Vicente Miquel Molina, Volume estimates for real hypersurfaces of a Kaehler manifold with strictly positive holomorphic sectional and antiholomorphic Ricci curvatures $\ldots \ldots \ldots \ldots \ldots \ldots 23$

Richard J. Griego and Andrzej Korzeniowski, Asymptotics for certain Wiener integrals associated with higher order differential operators

Abdeslam Mesnaoui, Unitary bordism of classifying spaces of quaternion

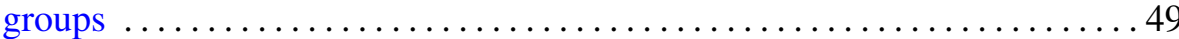

Abdeslam Mesnaoui, Unitary cobordism of classifying spaces of quaternion

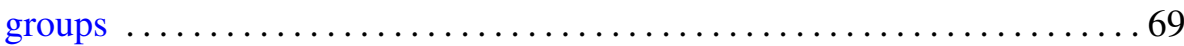

Jesper M. Møller, On equivariant function spaces $\ldots \ldots \ldots \ldots \ldots \ldots \ldots \ldots$

Bassam Nassrallah, A $q$-analogue of Appell's $F_{1}$ function, its integral representation and transformations

Peter A Ohring, Solvability of invariant differential operators on metabelian

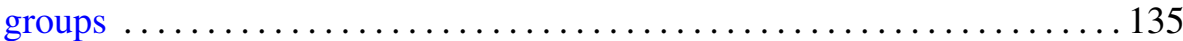

Athanase Papadopoulos and R. C. Penner, Enumerating pseudo-Anosov foliations

Ti-Jun Xiao and Liang Jin, On complete second order linear differential

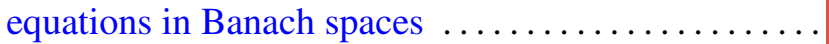

Carl Widland and Robert F. Lax, Weierstrass points on Gorenstein curves 